\title{
Simbiontes associados com Anomalocardia brasiliana (Gmelin) (Mollusca, Bivalvia, Veneridae) na llha de Santa Catarina e região continental adjacente, Santa Catarina, Brasil
}

\author{
Guisla Boehs ${ }^{1,2}$ \& Aimê Rachel M. Magalhães ${ }^{1}$ \\ ${ }^{1}$ Departamento de Aqüicultura, Centro de Ciências Agrárias, Universidade Federal de Santa Catarina. Rodovia Admar Gonzaga \\ 1346, Itacorubi,88040-900 Florianópolis, Santa Catarina, Brasil. E-mail: guislaboehs@hotmail.com; rachel@cca.ufsc.br \\ 2 Bolsista do CNPq, Pesquisa.
}

\begin{abstract}
Symbionts associated with Anomalocardia brasiliana (Gmelin) (Mollusca, Bivalvia, Veneridae) on Santa Catarina Island and adjacent continental region, Santa Catarina, Brazil. Pointed venus, Anomalocardia brasiliana (Gmelin, 1791), from natural beds of Santa Catarina Island and adjacent continental region (SE Brazil) were examined in respect of symbiotic associations. Holothuriophilus tomentosus (Ortmann, 1894) (Brachyura), Sphenia antillensis Dall \& Simpson, 1901 (Bivalvia), and polychaete worms (Polychaeta) were found by macroscopic diagnosis. By analysis of histological sections, it was noted trematode sporocysts (Digenea), a metacestode (Cestoda) and two ciliates (Ciliophora).

KEY WORDS. Commensalism, heterospecific relationships, marine molluscs, parasitism, symbiosis.
\end{abstract}

RESUMO. Berbigões, Anomalocardia brasiliana (Gmelin, 1791), de bancos naturais da Ilha de Santa Catarina e região continental adjacente (SC, Brasil), foram examinados quanto a presença de simbiontes. Holothuriophilus tomentosus (Ortmann, 1894) (Brachyura), Sphenia antillensis Dall \& Simpson, 1901 (Bivalvia) e poliquetos espionídeos (Polychaeta) foram observados macroscopicamente. A análise das seç̧ões histológicas evidenciou esporocistos de trematódeos (Digenea), um metacestóide (Cestoda) e dois ciliados (Ciliophora).

PALAVRAS CHAVE. Comensalismo, moluscos marinhos, parasitismo, relações heteroespecíficas, simbiose.

A simbiose é um termo utilizado para descrever associações heteroespecíficas onde há contato físico ou proximidade estreita entre duas espécies e inclui o parasitismo, o mutualismo, o comensalismo e a forese (CHENG 1967). Os critérios utilizados para definir, assim como para distinguir essas categorias entre si, têm sido objeto de discussão. Por exemplo, em um trabalho de revisão, Figueras \& Fisher (1988) levaram em conta aspectos de explotação, danos ao hospedeiro e dependência genética para distinguir o parasitismo do comensalismo. Por outro lado, consideraram a possibilidade da existência de um continuum entre os dois tipos de associação, o equilíbrio dependendo de fatores como o estado fisiológico de ambos os simbiontes, a defesa do hospedeiro e o estresse ambiental. CHENG (1967) definiu o comensalismo como uma relação heteroespecífica sem dependência metabólica, com obtenção de proteção física e de alimentos associados ao hospedeiro (mas que não fazem parte deste), ao passo que o parasitismo como uma relação com dependência metabólica do parasito em relação ao hospedeiro, seja em forma de material nutricional, enzimas digestivas, estímulo para o desenvolvimento ou controle da maturação. ANDERSON \& MAY (1978) consideraram três condições necessárias para classificar uma espécie animal como parasito: (1) utili- zar o hospedeiro como habitat, (2) depender nutricionalmente do mesmo e (3) lhe causar danos.

Danos significativos podem levar o hospedeiro ao estado de enfermidade. Esta foi definida por KINNE (1983) como um desvio negativo, estrutural e/ou funcional, do estado normal (de saúde) de um organismo, com redução do seu potencial ecológico em termos de sobrevivência, crescimento, reprodução, obtenção de energia, resistência ao estresse e capacidade de competição. Segundo KINNE (1983), nos bivalves marinhos, os principais agentes biológicos causadores de enfermidades pertencem ao grupo dos vírus, bactérias, fungos, Labyrinthomorpha, Apicomplexa, Ascetospora, Ciliophora, Trematoda Digenea, Annelida e Copepoda.

O berbigão Anomalocardia brasiliana distribui-se desde as Índias Ocidentais até o Uruguai, ocorrendo ao longo de toda a costa brasileira (Rıos 1994). Habita áreas protegidas da ação de ondas e de correntes, tanto na faixa entremarés como no infralitoral raso, onde enterra-se superficialmente no substrato lodoso ou areno-lodoso. É utilizado na alimentação humana e tem potencial para o cultivo. Na Ilha de Santa Catarina e região continental adjacente, onde forma extensos bancos, é expressivamente explotado e comercializado. 
Poucos são os registros na literatura sobre associações simbióticas com A. brasiliana. NARCHI (1966) relatou o encontro de Bucephalopsis haimeana (Lacaze-Duthiers, 1854) (Trematoda, Bucephalidae) em exemplares coletados no litoral do Estado de São Paulo. Também NARCHI (1972) registrou outros organismos associados com a espécie, como cracas (Crustacea, Cirripedia) e ovos de Nassarius Duméril, 1806 (Gastropoda, Nassariidae), como epibiontes, aderidos na face externa posterior da concha, parte exposta do animal quando este se enterra no sedimento. Adicionalmente, observou ainda casos esporádicos de poliquetos Polydora Bosc, 1802 (Spionidae). Melo \& Boens (2004) fizeram uma recente descrição do macho e redescrição da fêmea do caranguejo Pinnaxodes tomentosus Ortmann, 1894 (Pinnotheridae), a partir de exemplares associados com espécimens de $A$. brasiliana da Ilha de Santa Catarina (SC) e sugeriram a transferência dessa espécie de pinoterídeo para o gênero Holothuriophilus Nauk, 1880.

O presente trabalho relata os organismos associados em simbiose com A. brasiliana, incluindo parasitos e comensais, em pontos da Ilha de Santa Catarina e região continental adjacente (Santa Catarina, Brasil).

\section{MATERIAL E MÉTODOS}

Os berbigões foram coletados em dois locais da Ilha de Santa Catarina, denominados de Estação (Est.) I (Enseada de Ratones, Baía Norte, $27^{\circ} 27^{\prime} \mathrm{S}, 48^{\circ} 32^{\prime} \mathrm{W}$ ) e Est. II (Ribeirão da Ilha, Baía Sul, $\left.27^{\circ} 42^{\prime} \mathrm{S}, 48^{\circ} 33^{\prime} \mathrm{W}\right)$. Também foram analisados animais da Barra do Aririú, Baía Sul-continente $\left(27^{\circ} 43^{\prime} \mathrm{S}\right.$; $48^{\circ} 38^{\prime} \mathrm{W}$ ), ponto de coleta que foi denominado de Est. III.

No estudo foram utilizados 630 animais (Lote A), extraídos manualmente no entremarés e no infralitoral raso, entre maio de 2001 e maio de 2002. Adicionalmente, coletou-se animais, em igual número $(\mathrm{n}=30)$, para o cálculo de índices de condição (Lote $B, n=600$ ). A cada coleta, foi medida a temperatura e a salinidade da água dos locais, utilizando-se, respectivamente, um termômetro mercúrio padrão e um refratômetro manual. Em laboratório, os animais foram medidos quanto ao comprimento da concha (eixo ântero-posterior) e eviscerados. As amostras incluiram animais entre $17,2 \mathrm{~mm}$ e $41,1 \mathrm{~mm}$ de comprimento de concha (Média: 31,8 $\mathrm{mm} \pm 4,3$ ). Após o exame macroscópico, a carne de cada animal do Lote A foi fixada em solução de Davidson (Shaw \& Batrle 1957) por 24-48 h e depois transferida para álcool $70 \%$. Sequencialmente, cortes transversais foram feitos para a obtenção simultânea de amostras de tecidos do pé, manto, brânquias, tubo digestivo, gônada e glândula digestiva. Os tecidos fixados foram preparados de acordo com técnicas de histologia clássica, com inclusão em parafina, secções tissulares de aproximadamente $7 \mu \mathrm{m}$ e coloração por hematoxilina de Harris e eosina (HE). Os cortes histológicos foram examinados em microscopia de luz.

Os animais do Lote $\mathrm{B}$, após os procedimentos de obtenção de medida da concha, exame macroscópico quanto à presença de simbiontes e evisceração, foram mantidos em estufa, a $80^{\circ} \mathrm{C}$, até peso constante, para a obtenção do peso seco. Com esses dados, calculou-se o Índice de Condição: IC $(\%)=$ valor do peso da carne (g)/valor do peso da concha (g) x 100 (Cishell: RAINER \& MANN 1992).

\section{RESULTADOS E DISCUSSÃO}

A variação da temperatura e da salinidade, assim como os organismos associados com Anomalocardia brasiliana em cada local, estão sumarizados na tabela I.

Pinoterídeos Holothuriophilus tomentosus (Ortmann, 1894) foram observados vivos na cavidade palial de três berbigões $(33,2$ $36,2 \mathrm{~mm}$ de comprimento de concha), um em cada hospedeiro, na coleta de agosto de 2001, Lote B, na Enseada de Ratones (Est. I). A caracterização morfológica desses exemplares, assim como a revisão de aspectos taxonômicos, foram efetuadas por MeLo \& Boens (2004). A média do Índice de Condição (IC) dos berbigões desse local foi de $3,5 \%$ (DP $\pm 0,9 ; n=270$ ). O IC dos berbigões contendo os pinoterídeos foi de 1,4, 2,6 e 3,5\%. A partir desses resultados, observa-se que pelo menos um dos exemplares apresentou condição bastante abaixo da média obtida para a população. Esse aspecto reporta a uma discussão sobre o tipo de associação que esses crustáceos estabelecem com os moluscos bivalves, ou seja, se causam ou não impactos negativos significativos aos seus hospedeiros. Os pinoterídeos são amplamente conhecidos por sua associação com os moluscos bivalves, geralmente considerada como comensalismo. LAUCKNER (1983) relatou condições de enfermidade associadas com a presença desses organismos na cavidade do manto, incluindo perda de peso, redução da capacidade de filtração e danos físicos causados às brânquias, aos palpos e ao manto.

Um exemplar de Sphenia antillensis Dall \& Simpson, 1901 (Myidae) com 5,9 mm de comprimento de concha, foi observado na cavidade palial de um macho de A. brasiliana (34,6 $\mathrm{mm}$ de comprimento de concha), coletado em outubro de 2001, na Est. I (Lote A). Embora não haja registro anterior da ocorrência dessa espécie em A. brasiliana, Rios (1994) relatou a sua ocorrência em ostras (Bivalvia, Ostreidae) e em mexilhões (Bivalvia, Mytilidae). Na Enseada de Ratones, Boens et al. (2003) verificaram $S$. antillensis entre as raízes da marisma Spartina alterniflora Loiseleur, 1807.

Embora a presente associação parece representar um caso acidental (uma vez que uma única associação ocorreu em 570 berbigões analisados na Est. I e nenhum caso foi registrado nos outros locais de coleta), a localização da $S$. antillensis na cavidade palial de $A$. brasiliana, poderia ser um caso de comensalismo, já que há informação pretérita de sua associação com outros bivalves.

Poliquetos espionídeos, muito provavelmente do gênero Polydora (Spionidae), ocorreram em baixa frequência (em torno de $1 \%$ ) e intensidade de infestação nas valvas dos berbigões dos três locais. Os poliquetos espionídeos têm considerável importância econômica nos cultivos de ostras e de mexilhões (LaUCKNER 1983). Em A. brasiliana, conforme já observado por NARCHI (1972), a infestação por esses anelídeos é

Revista Brasileira de Zoologia 21 (4): 865-869, dezembro 2004 
Tabela I. Variação da temperatura e da salinidade da água nos pontos de coleta e organismos associados com Anomalocardia brasiliana: Estação I-Enseada de Ratones, Baía Norte; Estação II-Ribeirão da Ilha, Baía Sul (Ilha de Santa Catarina, Florianópolis, Santa Catarina) e Estação III-Barra do Aririú, Baía Sul-continente (Palhoça, Santa Catarina). (•) Prevalência abaixo de $1 \%$ e (••) entre 5 e $10 \%$, do total de animais analisados de cada local.

\begin{tabular}{|c|c|c|c|}
\hline & Estação I-Enseada de Ratone ${ }^{1}$ & Estação II-Ribeirão da Ilha ${ }^{1}$ & Estação III-Barra do Aririú ${ }^{2}$ \\
\hline Temperatura $\left({ }^{\circ} \mathrm{C}\right)$ & $16-29,5$ & $18-26,5$ & $19-22$ \\
\hline Salinidade (\%o) & $26-32,0$ & $18-34,0$ & $22-27$ \\
\hline Holothuriophilus tomentosus * & $\bullet$ & & \\
\hline Sphenia antillensis * & • & & \\
\hline Spionidae * & $\bullet$ & $\bullet$ & • \\
\hline Trematódeo digenético ** & $\bullet$ & & $\bullet$ \\
\hline Cestoda ** & & $\bullet$ & \\
\hline Ciliophora ${ }^{1 * *}$ & & & $\bullet$ \\
\hline Ciliophora $2 * *$ & $\bullet$ & & \\
\hline
\end{tabular}

${ }^{1}$ Período maio de 2001 a maio de 2002 (Estação I: 10 amostragens, Estação II: 7 amostragens); ${ }^{2}$ Período maio a setembro de 2001 (quatro amostragens); (*) diagnose macroscópica - Lotes A e B $(n=1.230) ;\left(^{* *}\right)$ resultados da histologia, Lote A $(n=630)$.

esporádica e, portanto, parece não representar risco significativo para indivíduos e populações.

Em 8,6\% dos berbigões da Est. I-Enseada de Ratones e em 5\% dos berbigões da Est. III-Barra do Aririú, observou-se esporocistos contendo massas germinativas e cercárias de um trematódeo digenético (Fig. 1). A prevalência mais alta (33\%) foi observada na Est. I, no mês de maio de 2001. Os esporocistos foram observados principalmente na área da gônada e entre os ácinos da glândula digestiva. Em cerca de 30\% dos indivíduos infestados observou-se castração parasitária.

Dentre os metazoários, os trematódeos digenéticos são os mais frequentes e considerados os mais importantes parasitos de bivalves (Kinne 1983). Espécies das famílias Fellodistomidae Nicoll, 1909 e Bucephalidae LaRue, 1926 causam castração parasitária e enfraquecimento do músculo adutor (PERKINS 1993). No berbigão A. brasiliana, o único relato pretérito de platielmintos larvais é o de NArCHI (1966), que identificou cercárias de Bucephalopsis haimeana (Bucephalidae) em espécimens procedentes do Mar Pequeno, São Vicente, Estado de São Paulo. Adicionalmente, bucefalídeos larvais do gênero Bucephalus von Baer, 1827 foram observados, no litoral brasileiro, em mexilhões Perna perna (Linnaeus, 1758) (Mytilidae) (Umiji et al. 1976, Lima et al. 2001, Silva et al. 2002) e em ostras Crassostrea rhizophorae (Guilding, 1828) (NASCIMENTO et al. 1986).

Um metacestóide encistado foi observado entre os ácinos da glândula digestiva de um indivíduo macho, com 23,4 mm de comprimento de concha, proveniente da coleta de maio de 2002, Est. II. Em volta do parasito, observou-se intensa reação hemocitária e uma cápsula fibrosa, formada pelo hospedeiro (Fig. 2). De acordo com observações anteriores em outras espécies de moluscos bivalves (Sindermann 1970, LaUCKNER 1983, Nascimento et al. 1986, Figueras \& Villalba 1988), esse cestóide pertence, muito provavelmente, ao gênero Tylocephalum Linton, 1890 (Lecanicephalidea, Tetragonocephalidae).
Larvas do gênero Tylocephalum parasitam diversos bivalves marinhos, particularmente ostras Crassostrea Sacco, 1897 e ostras perlíferas do gênero Pinctada Röding, 1798 (Bivalvia, Pteriidae), nas quais podem ocorrer em alta prevalência e intensidade de infestação, sendo que os adultos parasitam elasmobrânquios (LAUCKNER 1983). No Brasil, esses parasitos foram observados em ostras C. rhizophorae (NASCIMENTo et al. 1986). A sua localização na glândula digestiva coincide com os relatos de Figueras \& Villalba (1988) e de Lauckner (1983). No caso de ostras, esse último autor cita a ocorrência dessas larvas também nas brânquias.

Um ciliado foi observado no interior do tubo digestivo de um berbigão com 30,3 mm de comprimento de concha, fêmea, proveniente da coleta de setembro de 2002, na Est. III (Fig. 3). Trata-se de um ciliado do gênero Trichodina Ehrenberg, 1830 (Oligohymenophorea, Peritrichia, Mobilida). Espécies desse gênero estão relatadas como patogênicas para alguns bivalves e inócuas para outros (LAUCKNER 1983, Bower et al. 1994, CARbaLlaL et al. 2001). Em ostras do gênero Crassostrea, esses organismos produzem lesões no epitélio branquial e interferem no funcionamento normal das brânquias (Figueras \& Villalba 1988). No caso de C. gigas (Thunberg, 1793) severamente infestadas por uma espécie de Trichodina, Boussaid et al. (1999) observaram resposta inflamatória nas brânquias. BowER et al. (1994) relataram que em ostras do gênero Crassostrea, além de se localizarem nos filamentos branquiais, esses ciliados podem também ocorrer nos palpos labiais, no manto e na cavidade do manto.

Alguns exemplares de um outro ciliado (Ciliophora) foram observados na cavidade palial de um exemplar de $A$. brasiliana com 31,2 $\mathrm{mm}$ de comprimento de concha, coletado em maio de 2002, na Est. I (Fig. 4). De acordo com Kinne (1983), a maioria dos numerosos ciliados associados com bivalves marinhos é comensal, captando partículas alimentares nas brânquias e no epitélio do manto. Entretanto, LAUCKNER (1983) 

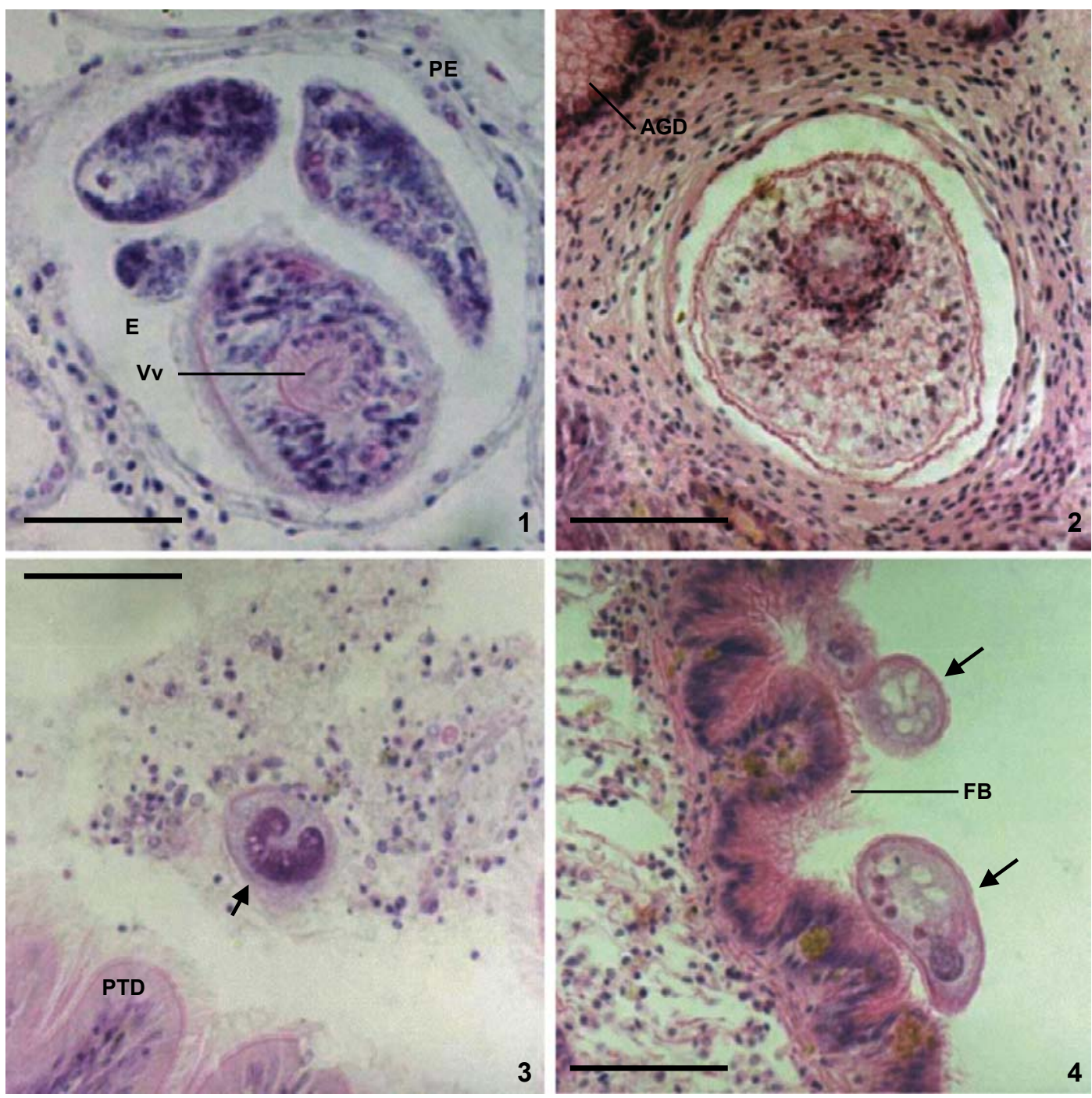

Figuras 1-4. Secções histológicas de berbigões Anomalocardia brasiliana provenientes da llha de Santa Catarina e região continental adjacente (Santa Catarina, Brasil), mostrando os organismos associados: (1) Cercárias de trematódeo digenético em diferentes fases de desenvolvimento, no interior de esporocisto, região da gônada; (2) metazoário encapsulado (Cestoda) na região da glândula digestiva; (3) Ciliado do gênero Trichodina, indicado por seta, no interior do tubo digestivo, (4) Ciliados, indicados por setas, na cavidade palial, próximo às brânquias. (AGD) Ácino da glândula digestiva, (E) esporocisto, (FB) filamento branquial, (PE) parede do esporocisto, (PTD) parede do tubo digestivo, ( $\mathrm{V} v$ ) ventosa ventral. Barras: $50 \mu \mathrm{m}$. Coloração: Hematoxilina-Eosina (HE).

considerou que quando presentes em grande número, ou em situações de adversidade ambiental e/ou estresse fisiológico do bivalve, esses simbiontes podem causar prejuízo ao seu hospedeiro. Conforme Figueras \& Villalba (1988), é, de fato, difícil estabelecer se esses protozoários atuam como comensais ou parasitos dos moluscos.

Partindo-se das premissas de ANDERson \& MAY (1978) para classificar um organismo como parasito (habitar, depender nutricionalmente e causar danos ao hospedeiro), dentre os or- ganismos observados em associação simbiótica com $A$. brasiliana no presente estudo, talvez se deva considerar apenas os platielmintos como casos seguros de parasitismo. No caso do cestóide, embora este tenha sido observado em baixa prevalência e intensidade de infestação, o dano potencial desse parasito ficou evidenciado pela intensa reação hemocitária do hospedeiro. No caso do trematódeo, tanto as prevalências altas (e.g. 33\%, Est. I, maio de 2001) quanto a castração parasitária (evidenciada em parte dos espécimens infestados), supos-

Revista Brasileira de Zoologia 21 (4): 865-869, dezembro 2004 
tamente representam risco para a população. Na avaliação de ANDERSON \& MAY (1978), parasitos, de modo geral, podem ter um importante papel no controle do crescimento populacional do hospedeiro, seja por causarem mortalidade e/ou por reduzirem o seu potencial reprodutivo.

\section{AGRADECIMENTOS}

Os autores são gratos ao CNPq pela concessão de bolsa para pesquisa ao primeiro autor (Processo 301341/00-1) e auxílio financeiro ao projeto (Processo 420075/01-1); ao Laboratório de Cultivo de Moluscos-Sambaqui e ao Depto. de Aqüicultura da UFSC, pelo apoio logístico; à Marilena A.A. Campos e Simone Carpes-Paternoster, pelo auxílio no processamento histotécnico; ao Dr. Antonio Villalba (CIMA, Xunta de Galicia, Espanha) e à Dra. Susan M. Bower (Fisheries and Oceans Canada, Pacific Biological Station), pelos valiosos comentários na fase de identificação de parte do material biológico.

\section{REFERÊNCIAS BIBLIOGRÁFICAS}

Anderson, R.M. \& R.M. May. 1978. Regulation and stability of host-parasite population interactions. Journal of Animal Ecology, London, 47: 219-247.

Boehs, G.; A. Blankensteyn; R. Alves; R.C. Sabry; F.G. Carvalho; J.A.S. Domingos \& J.W. Carvalho-Filho. 2003. Macrofauna bêntica de uma planície de maré da Enseada de Ratones, Ilha de Santa Catarina, SC, Brasil. Biotemas, Florianópolis, 16: 45-65.

Boussaid, B.; J.L. Grippari; T. Renault; G. Tige \& G. Dorange. 1999. Trichodina sp. infestation of Crassostrea gigas oyster gills in Brittany, France. Journal of Invertebrate Pathology, San Diego, 73: 339-342.

Bower, S.M.; S.E. McGladdery \& I.M. Price. 1994. Synopsis of infectious diseases and parasites of commercially exploited shellfish. Annual Review of Fish Diseases, Amsterdam, Elsevier, 4: 1-199.

Carballal, M.J.; D. Iglesias; J. Santamarina; B. Ferro-Soto \& A. Villalba. 2001. Parasites and pathologic conditions of the cockle Cerastoderma edule populations of the Coast of Galicia (NW Spain). Journal of Invertebrate Pathology, San Diego, 78: 87-97.

Cheng, T.C. 1967. Marine molluscs as hosts for symbioses with a review of known parasites of commercially important species, p. 1-424. In: F.S. Russell (Ed.). Advances in marine biology. London, Academic Press, vol. 5, XIII+424p.

Figueras, A.J. \& W.S. Fisher. 1988. Ecology and evolution of bivalve parasites. American Fisheries Society Special Publication, American Fisheries Society, Maryland, Virginia, 18: 130-137.

Figueras, A.J. \& A. Villalba. 1988. Patología de moluscos, p. $327-$ 389. In: J.E. Monteros \& U. Labarta (Eds). Patología en acuicultura. Madrid, FEUGA/Mundi-Prensa Libros, $\mathrm{XI}+551 \mathrm{p}$.
KINNE, O. 1983. Diseases of marine animals. Hamburg, Biologische Anstalt Helgoland, VIII+1028p.

LAUCKNER, G. 1983. Diseases of Mollusca: Bivalvia, p. 477-879. In: O. KInNE (Ed.). Diseases of marine animals. Hamburg, Biologische Anstalt Helgoland, vol. 2, VIII+1028p.

Lima, F.C.; M.G. Abreu \& E.F.M. Mesquita. 2001. Monitoramento histopatológico de mexilhão Perna perna da Lagoa de Itaipu, Niterói, RJ. Arquivo Brasileiro de Medicina Veterinária e Zootecnia. Belo Horizonte, 53: 203-206.

Melo, G.A.S. \& G. Boens. 2004. Rediscovery of Holothuriophilus tomentosus (Ortmann, 1894) comb. nov. (Crustacea, Brachyura, Pinnotheridae) in the Brazilian Coast. Revista Brasileira de Zoologia, Curitiba, 21: 229-232.

NARCHI, W. 1966. Encontro de Bucephalopsis haimeana (LacazeDuthiers) no Brasil. Ciência e Cultura, São Paulo, 18: 22-24. . 1972. Comparative study of the functional morphology of Anomalocardia brasiliana (Gmelin, 1791) and Tivela mactroides (Born, 1778) (Bivalvia, Veneridae). Bulletin of Marine Science, Miami, 22: 644-670.

Nascimento, I.A.; D.H. Smith; F. Kern II \& S.A. Pereira. 1986. Pathological findings in Crassostrea rhizophorae from Todos os Santos Bay, Bahia, Brazil. Journal of Invertebrate Pathology, San Diego, 47: 340-349.

Perkins, F.O. 1993. Infectious diseases of Molluscs, p. 255-287. In: J.A. Couch \& J.W. Fournie (Eds). Advances in fisheries science: Pathobiology of marine and estuarine organisms. Boca Raton, CRC Press, 552p.

Rainer, J.S. \& R. MANn. 1992. A comparison of methods for calculating condition index in eastern oysters, Crassostrea virginica (Gmelin, 1791). Journal of Shellfish Research, New York, 11: 55-58.

Rios, E.C. 1994. Seashells of Brazil. Rio Grande, Editora da Fundação Universidade do Rio Grande, 492p.

ShaW, B.L. \& H.I. Battle. 1957. The gross and microscopic anatomy of the digestive tract of the oyster Crassostrea virginica (Gmelin). Canadian Journal of Zoology, Otawa, 35: 325-347.

Silva, P.M.; A.R.M. Magalhães \& M.A. BarRacco. 2002. Effects of Bucephalus sp. (Trematoda: Bucephalidae) on Perna perna mussels from a culture station in Ratones Grande Island, Brazil. Journal of Invertebrate Pathology, San Diego, 79: 154-162.

SindermanN, C.J. 1970. Principal diseases of marine fish and shellfish. New York, Academic Press, 369p.

UmijI, S.; J.E. LunEtTa \& R.M.V. Leonel. 1976. Infestation of the mussel Perna perna by digenetic trematodes of the Bucephalidae family, gen. Bucephalus. Anais da Academia Brasileira de Ciências, São Paulo, 47: 115-117.

Recebido em 15.III.2004; aceito em 05.XI.2004. 